\title{
A Study of Intercept Adjusted Markov Switching Vector Autoregressive Model in Economic Time Series Data
}

\author{
*Seuk Wai, Phoong, Mohd Tahir Ismail, Siok Kun Sek \\ University Science Malaysia, Penang, Malaysia \\ *wai_phoong@yahoo.com
}

\begin{abstract}
Commodity price always related to the movement of stock market index. However real economic time series data always exhibit nonlinear properties such as structural change, jumps or break in the series through time. Therefore, linear time series models are no longer suitable and Markov Switching Vector Autoregressive models which able to study the asymmetry and regime switching behavior of the data are used in the study. Intercept adjusted Markov Switching Vector Autoregressive (MSI-VAR) model is discuss and applied in the study to capture the smooth transition of the stock index changes from recession state to growth state. Results found that the dramatically changes from one state to another state are continuous smooth transition in both regimes. In addition, the 1-step prediction probability for the two regime Markov Switching model which act as the filtered probability to the actual probability of the variables is converged to the actual probability when undergo an intercept adjusted after a shift. This prove that MSI-VAR model is suitable to use in examine the changes of the economic model and able to provide significance, valid and reliable results. While oil price and gold price also proved that as a factor in affecting the stock exchange.
\end{abstract}

Keywords: Markov Switching, Intercept Adjusted, Stationary, Bear, Bull

\section{Introduction}

Nonlinear time series models became popular through the past two decades in economic and financial field. Markov switching (MS) models are a nonlinear model which commonly applied in economic and financial time series to analysis and predict the economy relationship and the implication of the economic theories in real world data due to the dramatically changes or breaks that happened in the last decade such as oil price shocks, Asian Financial Crisis in 1997 and Global Economy 2008. Although a piecewise linear model able provide a valid result when estimate the changes, that is split the data around the time of the changes and then estimate in separate models; but it is also likely to be wasteful of information. In economic, time series models, that exhibit regime switching behavior might be inadequate for describing the evolution. Thus, a regime switching models was introduced to solve this problem. Markov switching autoregressive model that proposed by Hamilton (1989) is one of the popular nonlinear time series model. The Hamilton model is then extended to Markov switching vector autoregressive model (MS-VAR) by Krolzig (1997). The MS-VAR model is a generalization of the basic finite order of VAR model and the general idea of the model is the observables time series vectors are depend on the unobserved regime variable.

The advantage of MS-VAR model is able to provide a systematic approach to deliver statistical methods form drawing the past regime shift information of the data. This model also can estimate consistently and efficiently of the parameters in model, detect the recent shifts and correct the vector autoregressive model at times when the regimes alters and finally. Moreover, MS-VAR model also can increase the reliability of analysis of the business cycle and provide useful information in the study of economic relationship due to its properties on detection the high-level regime. Thus the economic relationship model between the oil price (OP) and gold price (GP) effect on Malaysia (KLCI), Singapore (STI), Thailand (SETI) and Indonesia (JCI) stock market returns are investigate by using intercept adjusted after regime switching model. Oil price shocks that happened from the year 1973 to 1974 and from the year 1979 to 1980 were examples of the structural changes of oil price in the world wide economic and this brings in a profound impact to all the countries in the world especially for the oil producer countries. Many academics start to explore and analysis the relationship between oil price shocks and macroeconomic variables during the last two decades. While the extremely stock down in the world in October 1987, and the crisis economy that cause the extreme drop of 
the exchange rate and shares in Asia countries in the year 1997 also show a structural break of time series behavior.

Commodity prices such as gold and oil always relate to the movement of the stock market prices and exchange rates. Although oil and gold are limited reservation but oil belongs to a basic of energy sources, which affect the country's economy as states by Sauterm and Awerbuch (2003) and gold, is a popular investment. Both of these two commodity prices implies on the country's stock market prices. According to Hamilton and Lin (1996), oil price is the major effect of macro economy growth since there was a large decreasing in macro economy after the increasing of oil price due to the analysis from the year 1986 . While based on the capital market theory, when economic activity and asset values are in a high-risk decreasing condition, the future cost stream will increase. Sauterm and Awerbuch (2003) describes that, most of the oil price was increasing during the period 1945 - 1985. From the year 1986, oil prices movement show a pattern change that was a radically price changes on the volatility of real oil price. While the oil price shock that happened after the year 1986 was affecting the stock market prices and bring in a detrimental effect since a higher production cost due to a higher oil prices will cause a decreasing in the earnings and in economy activities. Besides that, changes of the oil prices also will effect on the price volatility. Therefore, an immediate decline of the real stock returns also may occur because of the oil shocks and this as the interest of the study to capture the nonlinear properties in economic data. This paper is organized as the following structural. Section 2 presents the previous studies that related to this study. Section 3 depicts the sample and data that will used to calculate the economic data. Section 4 proposes the methodology of the study. Section 5 discusses the results and discussion. Lastly, section 6 presents the conclusion and recommendation of this paper.

\section{Literature Review}

Markov switching vector autoregressive (MS-VAR) model that characterize on a non-linear data generating process as piecewise linear by restricting the process to be linear in each unobservable and discrete number of regime are introduced by Krolzig (1997) based on the Hamilton idea. The MS-VAR model is a generalization of the basic finite order of VAR model and the general idea of the model is the observables time series vectors are depend on the unobserved regime variable. Besides that, the main characteristic of the Markov Switching model assumption was to govern the unobservable realization of the regime by a discrete time and state of Markov stochastic process by using transition probabilities. Moreover, MS-VAR model can estimate and predict a time-varying problem when a shift in parameters occurs. In the empirical study, only some parameters will be conditioned on the state of the Markov chain, while the other parameters are regime invariant. Therefore, four types of parameter estimation will introduce in the MS-VAR models that were Markov-Switching Mean, Markov-Switching Intercept Term, Markov-Switching Autoregressive Parameters and Markov-Switching Heteroskedasticity. The advantages of MS-VAR model are able to provide a systematic approach to deliver statistical methods and the model also can estimate consistently and efficiently of the parameters in model, detect the recent shifts and correct the vector autoregressive model at times when the regimes alters and finally. Jones and Kaul (1996) were the first investigators in analysis the international stock market reaction to oil shocks by predicted the stock returns due to the current and future changes in real cash flows. In the finding of Jones and Kaul (1996), oil prices in Canada, Japan, United Kingdom and United State stock market show an overall negative relationship between oil prices and stock market prices. This can be seen on the finding that during the post war period, oil prices show a significant detrimental effect on each country's stock market.

Moreover, an augmented market model was developed by Faff and Brailsford (1999) in estimate the sensitivity of Austrian industry equity returns where the oil price factors and market returns are estimated and found that oil, gas and diversified resources was significantly positive sensitivity to the oil price factors but negative sensitivity on the paper and packaging, and transport industries. In addition, gold and solid fuels industries showed a minor impact on the oil price effect in Faff and Brailsford (1999) study. Sadorsky (1999) was extended the Hamilton (1996), Jones \& Kaul (1996) studies on the oil price shock impact in short-term interest rate, and real stock returns. By using the impulse response function and vector autoregressive model (VAR), oil price was recorded a significantly negative relationship with real stock returns and a negative impact on real stock returns on interest rates and industrial production in US economy. Afshar et al. (2008) then re-examined Sadorsky study on the dynamic relationship between oil price shocks and stock market 
returns. Afshar et al. (2008) agreed with Sadorsky finding on the effect of oil price shocks to the real exchange rate and concluded that stable oil price and exchange rate contributed to the stability of the stock returns. Besides that, there are numerous authors were used the MS-VAR model in their study such as Hondroyiannis and Papapetrou (2006) study the dynamic relationship between stock returns and inflation in Greece by using a three regime MS-VAR model to capture the structural breaks observed during the period. While in the financial field, Guo et al. (2011) and Arin and Spagnolo (2011) are used a multivariate two regime MS-VAR model in study the financial data. In addition, there were numerous economists and statisticians adopt different statistical methods to study and predict the economic growth in the world. While Markov Switching models which can modeling the nonlinearities of the data and capturing each points in the regime shift were introduced and then was widely applied in analysis the economic fluctuation in the world

\section{Methodology}

Sample and Data: This study is a quantitative research and the data is taken from DATASTREAM and then transform into the natural logarithms to examine the economic relationship between oil price and gold price effect on the selected countries. The sample size of the study is 270 observations that are from December 1989 until May 2012.

Augmented Dickey-Fuller (ADF) test is applied in test the properties of the time series data. The ADF test is similar to the Dickey-Fuller test that has the regression,

$$
\Delta x_{t}=\sum_{-1}^{k-1} \beta_{j} \Delta x_{t-j}+\varepsilon_{t}
$$

Where $\Delta$ is the difference operator, parameter of estimate $\beta=\left(\beta_{1}, \ldots, \beta_{k}\right)$ is estimate based on its t-statistic test and $\varepsilon_{t}$ is white noise (Cheung and Lai, 1995).

While the ADF t-statistic test is estimated by using the Least Square method and the formula of the ADF test is $A D F_{t}=t_{\beta=1}=\frac{\beta-1}{S E(\beta)}$, where the $S E$ is represent to the standard error in the test.

ADF test is a stationary test which act as a preliminary step in the study to check the series is linear or nonlinear since Markov Switching models is used for estimate the nonlinear time series. Then intercept adjusted after a shift Markov Switching Vector Autoregressive model (MSI-VAR) is applied in examine the relationship between oil price and gold price with stock market index movement. Intercept adjusted after a shift Markov Switching Vector Autoregressive model (MSI-VAR) is applied in examine the relationship between oil price and gold price effect with stock market index movement. Among variety specifications that introduced by Krolzig (1997, 1998, 2000, 2001), intercept adjusted after regime switching is choose to apply in this paper due to the advantage that able to study the smooth transition of the sequence movement in the economic model.

The general idea of the MS-VAR model is the $n$-dimensional vector time series vector, $y_{t}=\left(y_{1 t}, \ldots, y_{n t}\right)$ depend upon an unobserved regime variable, $s_{t} \in\{1, \ldots, n\}$ which represents the probability of being in a particular state to the switching mechanism in various states. The conditional probability density of the observed $y_{t}$ is given by

$$
P\left(\left(y_{t} \mid Y_{t-1}\right), s_{t}\right)=\left\{\begin{array}{c}
h\left(y_{t} \mid Y_{t-1}, \beta_{1}\right) \text { if } s_{t}=1 \\
\ldots \\
h\left(y_{t} \mid Y_{t-1}, \ddot{\beta_{n}}\right) \text { if } s_{t}=n
\end{array}\right.
$$

Where $\beta_{\mathrm{n}}$ is the VAR parameter vector with regime $n$ and $Y_{t-1}$ is the past information. Thus, the parameter vector, $\beta$ depend on the regime at time $t$.

In this study, a two regime intercept adjusted after regime shift model, MSI-VAR model is $y_{t}=v\left(s_{t}\right)+$ $A_{1}\left(y_{t-1}\right)+A_{2}\left(y_{t-2}\right)+u_{t}$; where $u_{t}=N I D\left(0, \sum\left(s_{t}\right)\right)$ and $v$ is intercept term and $A_{k}$ are parameter shift function. 


\section{Results and Discussion}

All the variables series in the economic model has unit root problem and able to transform stationary after taking first differencing process. Then a two regime intercept adjusted after regime switching Markov Switching Vector Autoregressive model with first integrated order are used to analysis the economic data.

\begin{tabular}{|c|c|c|c|c|c|c|}
\hline $\log$ & \multicolumn{6}{|c|}{2160.6033} \\
\hline Likelihood & \multicolumn{6}{|c|}{-15.5941} \\
\hline AIC & \multicolumn{6}{|c|}{-15.2119} \\
\hline HQ & \multicolumn{6}{|c|}{-14.6427} \\
\hline \multicolumn{7}{|l|}{ SC } \\
\hline & $\mathrm{OP}$ & GP & KLCI & STI & SETI & JCI \\
\hline Const $\left(s_{t}=1\right)$ & -0.028 & -0.023 & -0.191 & -0.138 & -0.192 & -0.155 \\
\hline Const $\left(s_{t}=2\right)$ & 0.003 & 0.007 & 0.016 & 0.014 & 0.012 & 0.009 \\
\hline$\sigma$ & 0.062 & 0.043 & 0.070 & 0.070 & 0.093 & 0.105 \\
\hline \multicolumn{7}{|c|}{ Matrix Of Transition Probabilities, $p_{i j}$} \\
\hline & \multicolumn{2}{|c|}{$s_{t}=1$} & & \multicolumn{2}{|l|}{$s_{t}=2$} & \\
\hline$s_{t}=1$ & \multicolumn{2}{|c|}{0.669} & & \multicolumn{2}{|l|}{0.331} & \\
\hline$s_{t}=2$ & & & & 0.982 & & \\
\hline \multicolumn{3}{|c|}{ Regime Properties } & \multicolumn{2}{|c|}{ Probability } & \multicolumn{2}{|l|}{ Duration } \\
\hline$s_{t}=1$ & \multicolumn{2}{|c|}{14.700} & \multicolumn{2}{|c|}{0.0528} & \multicolumn{2}{|l|}{3.02} \\
\hline$s_{t}=2$ & \multicolumn{2}{|c|}{253.300} & \multicolumn{2}{|c|}{0.9472} & \multicolumn{2}{|l|}{54.23} \\
\hline
\end{tabular}

LR = Likelihood Ratio linearity test

** indicates that the p-value is significant at $5 \%$ level

The role of OP, GP, KLCI, STI, SETI and JCI are assessed with the MS(2)-VAR(1) model to estimate the relationship of the economic model. In MSI(2)-VAR(1) model, first regime $\left(s_{t}=1\right)$ represented to depression state while second regime $\left(s_{t}=2\right)$ represented to growth regime state since regime 1 coefficients is smaller than regime 2. In economy, recession state also known as "Bear" market and growth state also known as "Bull" market. MSI(2)-VAR(1) model is an intercept adjusted model after a switch in regime with variant intercept. In regime 2, all the coefficient of the lag component are positive in MSI-VAR model and STI recorded the most been affected by the OP and GP; while SETI is the less influencing of commodity price effect among these four countries. However, Malaysia reported has the most positive constant in regime 2. Generally, it is a common misconception that the log-likelihood value must be negative and the AIC value is positive; but if the likelihood is derived from the probability density then it is reasonable for the value is exceed 1 or a positive log-likelihood, and hence has a negative AIC. Thus, the likelihood ratio test of MSI(2)VAR(1) model show that the intercept regime dependent MS(2)-VAR(1) model pass all the misspecification tests. Moreover, the growth state or regime 2 is the most prevalent regime when examine the regime properties in both regimes by using MSI-VAR model.

The transition probabilities for MSI(2)-VAR(1) model are $p_{11}=0.699$ and $p_{22}=0.982$ suggest that the second regime is the persistence state since the transition probability of the growth regime is higher than the recession regime. Furthermore, the computed transition probability, $\operatorname{Prob}\left(s_{t}=1 \mid s_{t}=2\right)=0.331$ reported that an increasing transition probability occurs from a crisis regime (regime 1 ) to the growth regime (regime 2) and can be summarized in the matrix form,

$P=\left[\begin{array}{ll}0.699 & 0.331 \\ 0.018 & 0.982\end{array}\right]$

where the transition probabilities from crisis state to growth state is higher than the transition probabilities from growth state to recession state or also means that the stock market index is recover slowly then depression. Moreover, the second regime is the dominant regime since 253 observations or $94.72 \%$ of the 
total sample sizes are recorded in the regime 2. This illustrates that the economic relationship model is an asymmetric business cycle.

Figure 1: Probabilities graph of MSI(2)-VAR(1) model

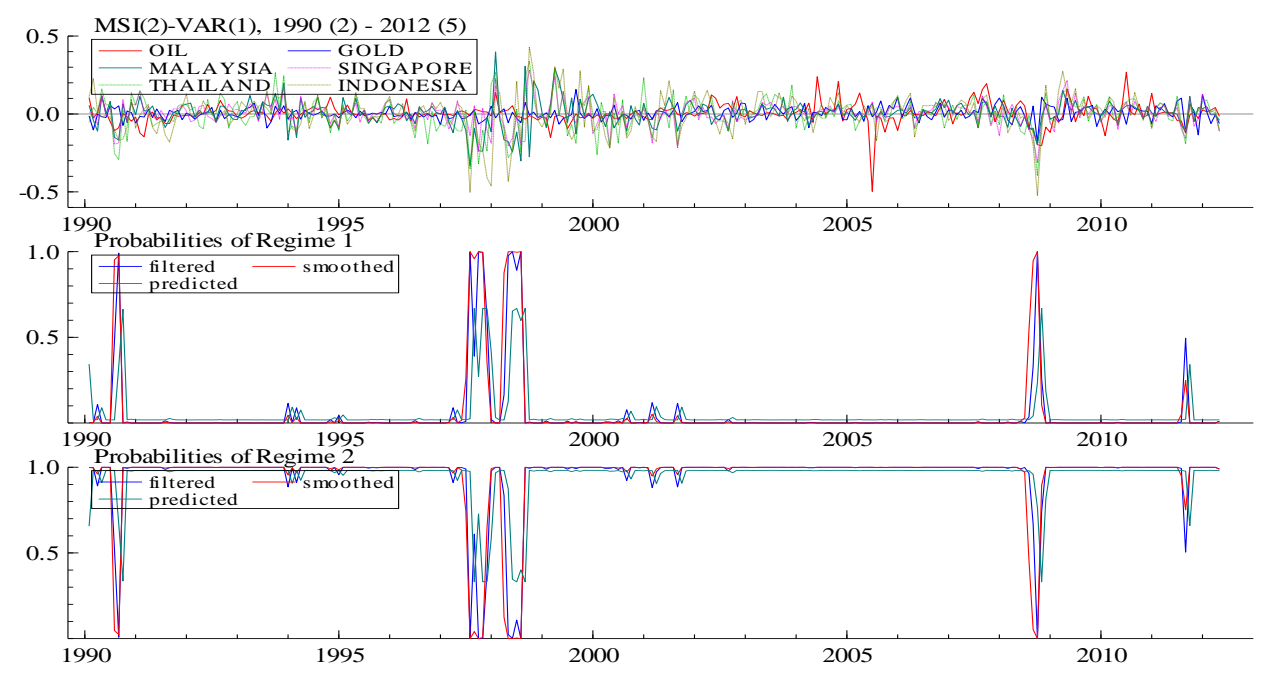

There are three panels reported in the graph when estimate the economic time series data by using MSI(2)VAR(1) model. The first panel in the graph presents the inferred regime probabilities of smooth transition of the probabilities into the intercept growth rate. Panel 2 and 3 depicts the filtered and smoothed probabilities of regime 1 and 2 which as the optimal inference of state variables and turning points in the economic model. Moreover, the filtered probabilities which is the optimal inference about the state variables by eliminate special components in the series are show in the panel 2 and 3 for the MSI-VAR model to explain the observable series data. While the continuous smooth transition in both regimes at panel 2 and 3 show the series do not has break but has structural change and jump in MSI(2)-VAR(1) model. In regime 1, structural changes of the series exist from August 1990 until September 1990, August 1997 until November 1997, April 1998 until August 1998 and from August 2008 until October 2008. In regime 2, there also got boom state from crisis region that are from February 1990 until July 1990, October 1990 until July 1997, September 1997 until March 1998 and from November 2008 until May 2012.

Figure 2: MSI (2)-VAR (1) model fit
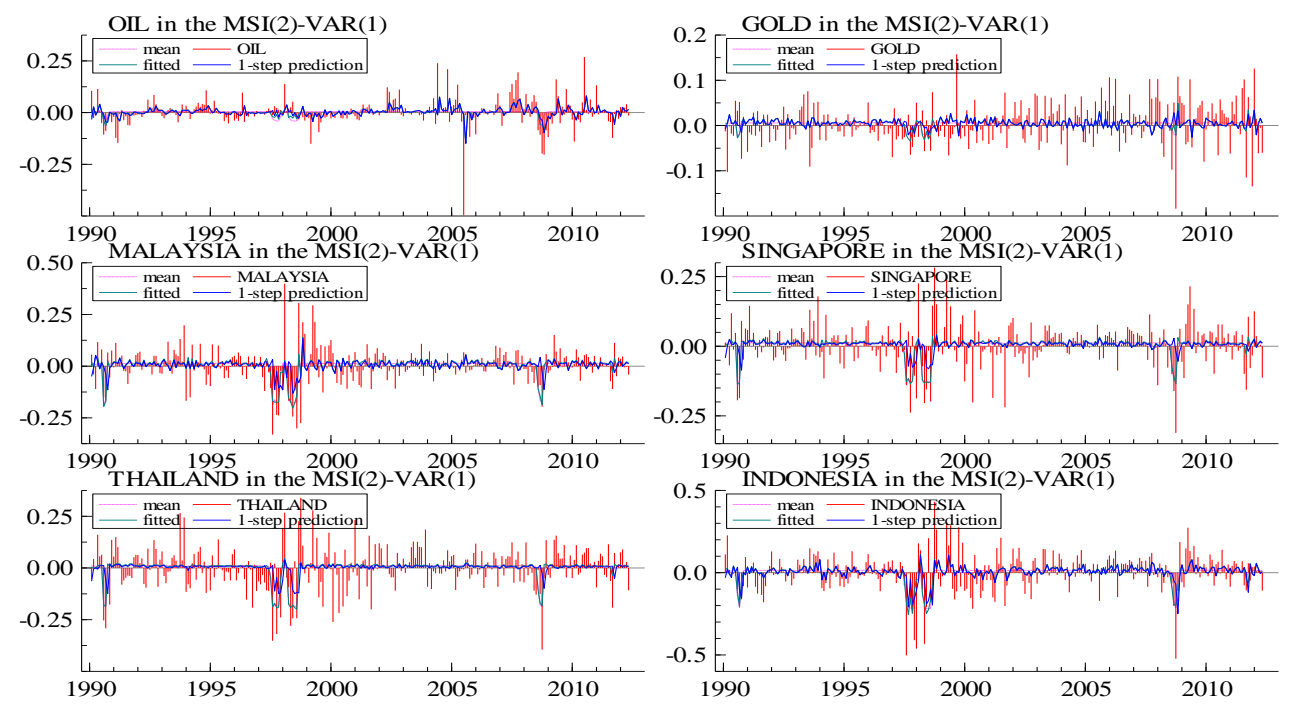
Figure 2 reported that the two regimes models are selected in estimate the model since the probabilities of the data is stick together when fit the predicted probabilities in more than two regimes. In addition, the 1step prediction probability for MSI(2)-VAR(1) model which act as the filtered probability to the actual probability of the variables is converged to the actual probability when intercept adjusted after a shift. This proved that MSI-VAR model is suitable to use in examine the changes of the economic model and able to provide significance, valid and reliable results.

\section{Conclusion and Recommendations}

ADF test is a stationary test in this study to examine the properties of the series in the economic model and results found that all the series are non-stationary. Thus, Markov switching models, which able to detect the structural change of the series are used in, estimate the variables in the economic model. Two regimes intercept adjusted Markov Switching Vector Autoregressive model with first integrated order is suitable to use in this study since the variables able to separate according to the state. While a decreasing of the oil price and gold price impact a depression of the Malaysia, Singapore, Thailand and Indonesia stock market returns. Therefore, oil price and gold price are concluded as the factors in affecting the stock market exchange. This can be supported that oil is a basic consumption for the transportation and gold is a popular investment, precious metal and jewelry, which are the facts that able to affect the share. Moreover, the MSI(2)-VAR(1) model able to provide a significant and reliable results since it able to fit the data series and capture the smooth transition of the time series data. Moreover, all data in this study are taken from DATASTREAM and analysis by using the statistical software packages Eviews and Ox. Besides that, further study can be carry out in studying the other commodity price or precious metal effect on stock exchange. While other statistical methods such as finite mixture model, Markov switching vector error correction model also can be explore to capture the transition of the economic time series data.

\section{References}

Afshar, T. A., Arabian, G. \& Zomorrodian, R. (2008). Oil price shocks and the US stock market. In: IABR \& TLC Conference Proceedings, 24-26 April 2008. USA. San Juan, Puerto Rico: The International Academy of Business and Public Administration Disciplines.

Arin, K. P. \& Spagnolo, N. (2011). Short-term growth effect of fiscal policy revisited: A Markov switching approach. Economic Letter, 110, 278-281.

Cheung, Y. W. \& Lai, K. S. (1995). Lag order and critical values of the augmented dickey-fuller test. Journal of Business \& Economic Statistic, 13(3), 277-280.

Faff, R. \& Brailsford, T. J. (1999). Oil price risk and the Australian stock market. Journal of Energy Finance and Development, 49, 69-87.

Guo, F., Chen, G. R. \& Huang, Y. S. (2011). Market contagion during financial crisis: A regime switching approach. International Review of Economics and Finance, 20, 95-109.

Hamilton, J. D. (1989). A new approach to the economic analysis of nonstationary time series and the business cycle. Econometrica, 57, 357-384.

Hamilton, J. D. \& Lin, G. (1996). Stock market volatility and the business cycles. Journal of Applied Econometrics, 11, 411-434.

Hondroyiannis, G. \& Papapetrou, E. (2006). Stock returns and inflation in Greece: A Markov switching approach. Review of Financial Economics, 15, 76-94.

Jones, C. M. \& Kaul, G. (1996). Oil and the stock markets. The Journal of Finance, 51, 463-491.

Krolzig, H. M. (1997). Markov-switching Vector Autoregression. Berlin: Springer.

Krolzig, H. M. (1998). Econometrics modeling of Markov switching vector autoregressions using MSVAR for Ox. Technique Report. Faculty Economy, University Oxford.

Krolzig, H. M. (2000). Predicting Markov-switching vector autoregressive process. Technique Report. Faculty Economy, University Oxford.

Krolzig, H. M. (2001). Markov switching procedures for dating the Euro-zone business cycle. Vierteljahreshefte zur Wirtschaftsforschung, 70, 339-351.

Kugler, P. (1996). The term structure of interest rates and regime shifts: Some empirical results. Economic Letters, 50, 121-126. 
Sauterm R. \& Awebuch, S. (2003). Oil price volatility and economic activity: a survey and literature review. IEA Research Paper, IEA, Paris, August 2003.

Sadorsky, P. (1999). Oil price shocks and stock market activity. Energy Economics, 21, 449-469. 